\title{
Transferencia Tecnológica en Universidades Chilenas: El Caso de la Universidad de Concepción
}

\author{
Pablo Catalán ${ }^{1 *}$ Eliana Sepúlveda ${ }^{1}$ Annabella Zapata ${ }^{1}$
}

\begin{abstract}
Resumen: El presente estudio busca contribuir a la identificación de los determinantes de transferencia tecnológica en la Universidad de Concepción (UDEC), universidad chilena de larga tradición en investigación aplicada. Con tal fin, se aplicó un modelo probit a una muestra de 190 proyectos de Investigación y Desarrollo (I+D) ejecutados por la universidad entre los años 2005 y 2016. De forma de observar en mayor detalle posibles tendencias temporales, la muestra fue dividida en dos períodos: 2005-2013 y 2014-2016. Para el período 2005-2016, los resultados muestran que el financiamiento público de I+D y el número de proyectos de I+D en los cuales ha participado el investigador principal inciden positivamente en la probabilidad de transferencia tecnológica, mientras que el número de publicaciones científicas asociadas al investigador principal presenta un efecto negativo. En cuanto a los subperíodos propuestos, en ambos casos el financiamiento público de I+D mantiene su efecto positivo. Adicionalmente, el número de patentes en el primer período, y el financiamiento privado de I+D y el número de organizaciones sociales que participan del proyecto en el segundo período se suman como determinantes de efecto positivo. Asimismo, durante el último período, el número de organismos públicos que participan en el proyecto y el número de publicaciones científicas que posee el investigador principal presentan efectos negativos en la probabilidad de transferencia tecnológica.
\end{abstract}

Keywords: transferencia tecnológica; universidades; investigación y desarrollo; capacidades de investigación

Title: Technology Transfer in Chilean Universities: The Case of The University of Concepcion

Abstract:The present study aims to identify the determinants of technology transfer in the University of Concepción (UDEC), a Chilean university with a long-applied research tradition. To address the research question we applied a probit model to a sample of $190 \mathrm{R} \& \mathrm{D}$ projects executed by UDEC researchers between 2005 and 2016. To observe in greater detail timing trends we divided the sample into two periods: 2005-2013 and 20142016. During the period 2005-2016, we found that R\&D public funding and the number of previous R\&D projects per principal investigator have a positive effect on the likelihood of technology transfer, whereas the number of scientific publication per principal investigator shows a negative effect. In terms of the sub periods proposed, the positive effect of $\mathrm{R} \& \mathrm{D}$ public funding remains in both cases. In addition, the number of patents per principal investigator during the first period, and $\mathrm{R} \& \mathrm{D}$ private funding and the number of social organizations per project during the second period positively affect the likelihood of technology transfer. Likewise, during the last period, the number of public organizations that participate in each $R \& D$ project and the number of publications per principal investigator has a negative impact on technology transfer.

Keywords: technology transfer; universities; research and development; research capacity

Submitted: Jan $18^{\text {th }}, 2019$ / Approved: Aug $26^{\text {th }}, 2019$

\section{Introducción}

Tradicionalmente, la justificación de las universidades se ha basado en la provisión de personas capacitadas, la preservación de la herencia cultural y el avance del conocimiento en las distintas ramas. Sin embargo, los temas económicos se han vuelto tan importantes como los culturales, lo cual ha provocado una ampliación del papel de las universidades en la sociedad frente a los distintos individuos, proyectando una nueva imagen de centros de innovación tecnológica y desarrollo económico regional (Peters, 1989). Así, las universidades han asumido un papel como inventor y agente de transferencia de conocimiento y tecnología (Etzkowitz, 2017). De este modo, en la actualidad se extiende la misión de las universidades a la solución de problemas y demandas de mediano y corto plazo del sector empresarial y de la sociedad en general, lo que ha exigido a las universidades una reconceptualización y reordenamiento organizativo para realizar los procesos de producción, almacenamiento y transferencia del conocimiento (López, Mejía, \& Schmal, 2006).

En economías emergentes como Chile, las capacidades de Investigación y Desarrollo (I+D) tienden a estar altamente concentradas dentro de universidades e institutos de investigación pública (Fernández, Otero, Rodeiro, \& Rodríguez, 2009), siendo necesarias políticas sistemáticas de transferencia tecnológica de modo de facilitar la fluidez de la relación entre el mundo académico y empresarial chileno. En Chile, se destina en promedio un $26 \%$ del presupuesto nacional para $\mathrm{I}+\mathrm{D}$ al financiamiento de investigación básica, mientras que un $41 \%$ se destina a investigación aplicada, dejando un 33\% para invertir en desarrollo experimental para actividades comerciales, con apenas un $0,4 \%$ del PIB nacional destinado a I+D.

(1) Departamento de Ingeniería Industrial, Universidad de Concepción, Chile.

*Autor de correspondencia: pacatala@udec.cl 
La presente investigación pretende aportar al desarrollo de políticas públicas que permitan mejorar los indicadores de transferencia tecnológica universitaria en Chile, a través de la exploración de las dinámicas de transferencia tecnológica en una universidad chilena compleja y de orientación pública, tomando como caso particular la Universidad de Concepción. Con tal fin, se realizó una investigación de carácter cuantitativo para la cual se recopilaron datos asociados a los proyectos de I+D desarrollados dentro de la universidad, luego se aplicó un modelo de respuesta binaria probit con el fin de identificar qué factores determinan que un proyecto transfiera efectivamente su conocimiento al mercado. Adicionalmente, mediante el mismo modelo, se realizó un análisis por período de tiempo para identificar si los determinantes de la transferencia tecnológica en la Universidad de Concepción se ven condicionados por el cierre temporal del proyecto. Lo anterior se realizó en base a cinco factores principales: 1) Financiamiento de I+D, 2) Capacidades de Investigación, 3) Estructuras de Soporte Tecnológico, 4) Redes y 5) Estructura de Soporte Académico. Es así como el objetivo del estudio es responder la pregunta ¿Cuáles son los factores que rigen la transferencia tecnológica en una universidad chilena compleja y de orientación pública?, pues la identificación de estos factores puede constituir nuevo conocimiento útil para la formulación de protocolos institucionales y políticas públicas por parte de hacedores de política y autoridades académicas, pudiendo resultar ello en una mejora de la gestión de la ciencia, tecnología e innovación en las universidades chilenas.

La presente investigación se estructura de la siguiente manera: luego de la introducción, se presenta el marco teórico de la investigación. Posteriormente, se describe la metodología utilizada en el desarrollo del estudio. A continuación, se muestran los resultados y análisis de la investigación y finalmente, se presentan las conclusiones de la investigación junto con las implicancias que los resultados obtenidos tienen para la formulación de futuras políticas públicas.

\section{Marco Teórico}

La transferencia tecnológica desde las universidades y centros de investigación hacia el sector privado ha cobrado cada vez más importancia dentro del contexto actual, de tal manera que los temas asociados a transferencia tecnológica se han convertido en una prioridad de las agendas políticas y académicas en distintos países del mundo (Rodriguez \& Casani, 2007). Tradicionalmente, las universidades han cumplido dos funciones dentro de la sociedad: educar a los estudiantes y realizar investigaciones. En los últimos años, estas instituciones han debido incorporar una función adicional: promover la comercialización de los resultados derivados de su investigación (Fernández et al., 2009).

La expansión generada en el rol de la universidad chilena, ha requerido grandes cambios en cuanto a políticas, distribución de recursos y cultura académica, derivando en una significativa reorganización del Sistema Nacional de Ciencia, Tecnología e Innovación (SNCTI). Este sistema está compuesto por entidades tanto públicas como privadas, cuya operación se orienta al desarrollo de investigación básica y aplicada, formación de capital humano, innovación y emprendimiento.
Para contribuir con el buen desempeño del SNCTI, la participación estatal se vuelve esencial. El gobierno chileno ha formulado políticas públicas de forma de apoyar la investigación e innovación que desarrollan las empresas y universidades chilenas a través de diferentes fondos e incentivos de financiamiento. Por un lado, ha invertido mediante las principales agencias del Sistema de Innovación Chileno, CONICYT y CORFO. Por otro lado, se han promovido programas de asociatividad ciencia-empresa mediante la ejecución de proyectos de I+D puntuales que funcionan bajo la modalidad de fondos concursables.

En general, la transferencia tecnológica de las universidades en Chile se ve condicionada por la acción del Estado en cuanto a la generación de normas que regulen de buena manera la propiedad intelectual, la promoción de la protección de invenciones y la puesta a disposición de la comunidad, la predominancia del financiamiento público en las actividades asociadas con I+D y transferencia tecnológica, como también en el fomento de la innovación y adopción de nueva tecnologías por parte del sector productivo.

El aumento en el fortalecimiento de los incentivos estatales hacia la innovación y el emprendimiento en los últimos años ha generado un incremento significativo del gasto en I+D por parte del gobierno chileno. Sin embargo, este aumento no ha sido suficiente para acercar a Chile a países con economías similares en términos de I+D (Lederman \& Maloney, 2014).

\section{Transferencia Tecnológica}

Dentro de la literatura existente, el concepto de transferencia tecnológica se aprecia como un concepto bastante amplio. Según la Association of University Technology Managers (AUTM), para la mayoría de las universidades y centros de investigación, la transferencia tecnológica se define como el proceso de transferir de una organización a otra los descubrimientos científicos, con el fin de promover el desarrollo y la comercialización ${ }^{1}$.

Existen diferentes canales y mecanismos para la transferencia tecnológica, asociados a las relaciones recíprocas entre universidad-industria-gobierno, a procesos de interacción universidad-empresa o a modelos de innovación abierta. Dentro de los canales de transferencia tecnológica universitaria destaca la literatura abierta, las patentes, derechos de autor, licencias, demostraciones personales, intercambios de personal, spin-offs (Bozeman, Rimes, \& Youtie, 2015). También son mencionados mecanismos de transferencia tecnológica menos renombrados, como las importaciones de bienes de capital, la inversión extranjera directa y el licenciamiento de tecnologías, estándares de productos y procesos entre productor y proveedor, contratación de nuevos graduados y posgraduados, programas de capacitación, publicaciones científicas, conferencias y redes de interacción informales entre científicos y empresas (Zuñiga \& Correa, 2013). Es importante destacar que el éxito de la transferencia tecnológica universitaria mediante los distintos métodos no termina cuando la tecnología es

${ }^{1}$ Association of University Technology Managers, 2017. Recuperado de https://www.autm.net/autm-info/about-tech-transfer 
comercializada y entregada a la industria, sino que requiere la utilización de la tecnología en nuevos productos, procesos o cambios organizativos innovadores (Heinzl, Kor, Orange, \& Kaufmann, 2013).

Dentro de los medios existentes, se considera que las patentes son el mecanismo más común que facilita protección de la propiedad intelectual y la posterior transferencia tecnológica por parte de las universidades (Van Norman \& Eisenkot, 2017). En tal sentido, el éxito de las patentes universitarias se ve sujeto a distintos factores. Azagra (2001) determinó que el financiamiento público es la variable más importante de cara a la generación de patentes, pero que existe sinergia con el financiamiento complementario de las empresas. También obtiene dentro de sus resultados una relación con el tamaño de los departamentos, afirmando que los departamentos universitarios de mayor tamaño, inmersos en la enseñanza de masas, muestran una menor propensión a patentar.

En los últimos años, las empresas nacidas dentro de los centros de investigación y universidades, categorizadas como spin-offs, se han convertido en uno de los mecanismos más eficaces de transferencia de resultados de investigación y tecnología, contribuyendo al desarrollo empresarial y potenciando un crecimiento económico que incide sobre la mejora competitiva del sector productivo en su conjunto (Iglesias, Jambrino, \& Peñafiel, 2012). El éxito de estas empresas es un tema de estudio recurrente en la literatura. Sternberg (2014) ilustra como el contexto que rodea a las spin-off, específicamente su entorno regional, afecta de manera significativa su éxito, mientras que, Beraza \& Rodríguez (2010) recalcan en su estudio que la transferencia tecnológica mediante spin-offs se ve favorecida por determinantes propios del inventor como su espíritu emprendedor, su experiencia profesional previa y su influencia personal en la empresa. En el ámbito financiero, Iglesias et al., (2012) concluye que las fuentes de apoyo económico que favorecen al desarrollo de la actividad de I+D de las spin-offs universitarias no son solo las ayudas con recursos públicos, sino que también adquiere relevancia la participación externa de capital, la modalidad de proyectos en colaboración y el ineludible desafío de dirigirse al mercado internacional.

La comercialización por los distintos medios de transferencia tecnológica también se ve determinada por la calidad de la investigación universitaria a nivel de departamentos, por la participación del investigador en el proceso y por la existencia de normas y procedimientos internos de apoyo. El tipo de universidad y su localización también son factores que afectan de manera relevante la comercialización, siendo las universidades privadas más propensas a responder a los cambios del entorno que las universidades estatales y, por ende, tienen mayores indicadores de transferencia tecnológica, mientras que las universidades localizadas en regiones, con concentración empresarial de alta tecnología, se ven potenciadas en su comercialización tecnológica (Perkmann et al., 2013; Thursby, Jensen, \& Thursby, 2001). La existencia de redes es uno de los mayores determinantes de la comercialización, siendo una de las relaciones más estudiadas la relación universidad-industria.
Los canales y mecanismos tratados que favorecen la comercialización de know-how tecnológico desde la universidad al mercado, se ven apoyados por instituciones que facilitan el proceso (Bradley, Hayter, \& Link, 2013). Estas instituciones pueden ser clasificadas en intermediarios internos, dentro de los cuales están las Oficinas de Transferencia y Licenciamiento (OTLs), Incubadoras y Parques Científicos, como también en intermediarios externos, dentro de los cuales se encuentran empresarios, firmas de capital de riesgo y agencias de desarrollo (Wright, Clarysse, Lockett, \& Knockaert, 2008).

Las OTLs tienen como objetivo comercializar resultados de investigación a través de patentes, licencias y empresas spin-off, facilitando la transferencia de conocimientos universitarios al mercado mediante distintas formas de propiedad intelectual que resulten de la investigación universitaria (Algieri, Aquino, \& Succurro, 2013; Siegel, Waldman, Atwater, \& Link, 2004). Para esto, se debe tener en consideración el potencial comercial de la invención, así como también, el interés existente en el sector público y privado (Siegel, Waldman, \& Link, 2003). En general, la capacidad de éxito en el apoyo a la comercialización que tienen estas instituciones se ve afectada por factores internos, dentro de los cuales se encuentra la edad de la OTL y el número de trabajadores que se desempeñen dentro de la institución (Foltz, Barham, \& Kim, 2000; Thursby et al., 2001). La presente investigación busca identificar cuáles son los factores, tanto internos como externos, que determinan las capacidades de transferencia tecnológica mediante los distintos mecanismos definidos a partir de una visión enfocada en la Universidad de Concepción en Chile.

\section{Metodología}

\section{Datos}

La base de la investigación fueron proyectos de Investigación y Desarrollo (I+D) ejecutados por la Universidad de Concepción entre los años 2005 y 2016. Se utilizó información contenida en la base de datos de la Vicerrectoría de Investigación y Desarrollo (VRID) de la universidad, de donde se obtuvieron datos asociados a los proyectos efectivamente realizados y cerrados durante los años de estudio y antecedentes básicos sobre los investigadores responsables del proyecto. A partir de la base de datos obtenida, se procedió a eliminar ciertos proyectos cuyo fin no era la transferencia tecnológica y otros de los cuales no se pudo obtener información precisa sobre los resultados finales del proyecto. Posterior a la corrección, se levantó información referente a campos adicionales necesarios para el testeo del modelo econométrico propuesto, obteniéndose una base de datos compuesta por un total de 190 proyectos de $\mathrm{I}+\mathrm{D}$.

A continuación, se procede a la descripción detallada de todas las variables incorporadas en la base de datos utilizada, tanto variable dependiente como variables explicativas. 
Tabla 1

Variables modelo de respuesta binaria probit

\begin{tabular}{|c|c|c|c|}
\hline Macro Variable & $\begin{array}{l}\text { Nombre de la variable } \\
\text { en el modelo }\end{array}$ & $\begin{array}{l}\text { Nombre de la variable } \\
\text { en software }\end{array}$ & Descripción de la variable \\
\hline $\begin{array}{l}\text { Transferencia Tecnológica } \\
\text { (dependiente) }\end{array}$ & $\begin{array}{l}\text { Transferencia Tecno- } \\
\text { lógica }\end{array}$ & $\begin{array}{l}\text { transferencia tecno- } \\
\text { lógica }\end{array}$ & $\begin{array}{l}\text { Variable dicotómica. Toma el valor } 1 \text { si el proyecto de } \mathrm{I}+\mathrm{D} \text { ha realizado transfe- } \\
\text { rencia de conocimiento por al menos uno de los siguientes mecanismos; acuer- } \\
\text { do de licencia, consultoría, contratos } \mathrm{I}+\mathrm{D} \text {, creación de empresas spin-off } \mathrm{y} / \mathrm{o} \\
\text { solicitud de patente.; } 0 \text { en caso contrario }\end{array}$ \\
\hline \multirow{2}{*}{$\begin{array}{l}\text { Financiamiento de I+D (ex- } \\
\text { plicativa) }\end{array}$} & $\begin{array}{l}\text { Financiamiento Públi- } \\
\text { co de I+D }\end{array}$ & $\begin{array}{l}\text { financiamiento } \\
\text { publico }\end{array}$ & $\begin{array}{l}\text { Variable explicativa continua. Indica el monto de financiamiento (millones de } \\
\text { pesos) que aportó la agencia pública asociada al proyecto de I+D analizado para } \\
\text { su desarrollo. }\end{array}$ \\
\hline & $\begin{array}{l}\text { Financiamiento Priva- } \\
\text { do de } \mathrm{I}+\mathrm{D}\end{array}$ & $\begin{array}{l}\text { financiamiento } \\
\text { privado }\end{array}$ & $\begin{array}{l}\text { Variable explicativa continua, que indica el monto de financiamiento pecunia- } \\
\text { rio que aportaron las empresas participantes en el proyecto de } \mathrm{I}+\mathrm{D} \text { analizado. }\end{array}$ \\
\hline \multirow{7}{*}{$\begin{array}{l}\text { Capacidades de Investiga- } \\
\text { ción (explicativa) }\end{array}$} & $\begin{array}{l}\text { Número de Académi- } \\
\text { cos en Proyecto }\end{array}$ & total académicos & $\begin{array}{l}\text { Variable explicativa discreta. Indica el número de académicos de la universidad } \\
\text { que participaron en el proyecto de } \mathrm{I}+\mathrm{D} \text { analizado. }\end{array}$ \\
\hline & $\begin{array}{l}\text { Número de Alumnos } \\
\text { en Proyecto }\end{array}$ & total alumnos & $\begin{array}{l}\text { Variable explicativa discreta. Indica el número de alumnos de pregrado y post- } \\
\text { grado que participaron en el proyecto de I+D muestreado. }\end{array}$ \\
\hline & $\begin{array}{l}\text { Cargo Director del } \\
\text { Proyecto }\end{array}$ & director centro $\mathrm{i}+\mathrm{d}$ & $\begin{array}{l}\text { Variable explicativa dicotómica. Toma el valor de } 1 \text { si el director del proyecto } \\
\text { de I+D dirige un Centro o Laboratorio I+D dentro de la universidad, } 0 \text { en caso } \\
\text { contrario. }\end{array}$ \\
\hline & $\begin{array}{l}\text { Número de Patentes } \\
\text { Solicitadas }\end{array}$ & $\mathrm{n}^{\circ}$ patentes solicitadas & $\begin{array}{l}\text { Variable explicativa discreta. Indica el número de patentes que tiene solicitadas } \\
\text { el director del proyecto de I+D analizado. }\end{array}$ \\
\hline & $\begin{array}{l}\text { Número de Patentes } \\
\text { Adjudicadas }\end{array}$ & $\begin{array}{l}\mathrm{n}^{\circ} \text { patentes adjudica- } \\
\text { das }\end{array}$ & $\begin{array}{l}\text { Variable explicativa discreta. Indica el número de patentes que tiene adjudica- } \\
\text { das el director del proyecto de } \mathrm{I}+\mathrm{D} \text { analizado. }\end{array}$ \\
\hline & $\begin{array}{l}\text { Número de Proyectos } \\
\text { de I+D }\end{array}$ & $\mathrm{n}^{\circ}$ proyectos & $\begin{array}{l}\text { Variable explicativa discreta. Indica el número de proyectos de } \mathrm{I}+\mathrm{D} \text { en que ha } \\
\text { participado el académico. }\end{array}$ \\
\hline & $\begin{array}{l}\text { Número de Publica- } \\
\text { ciones }\end{array}$ & $\mathrm{n}^{\circ}$ publicaciones & $\begin{array}{l}\text { Variable explicativa discreta. Indica el número de publicaciones ISI y Scielo que } \\
\text { tiene el director del proyecto de I+D analizado durante el período de estudio. }\end{array}$ \\
\hline $\begin{array}{l}\text { Estructura de Soporte Tec- } \\
\text { nológico (explicativa) }\end{array}$ & $\begin{array}{l}\text { Número Personal de } \\
\text { la OTL }\end{array}$ & $\mathrm{n}^{\circ}$ trabajadores otl & $\begin{array}{l}\text { Variable explicativa discreta. Indica el número de personas que trabajan en la } \\
\text { OTL de la universidad en el año de inicio de cada proyecto. Si la OTL no ex- } \\
\text { istía en la fecha de inicio del proyecto, se consideró un valor de cero personas. }\end{array}$ \\
\hline \multirow{4}{*}{ Redes (explicativa) } & $\begin{array}{l}\text { Número de Empresas } \\
\text { Asociadas }\end{array}$ & $\mathrm{n}^{\circ}$ empresas & $\begin{array}{l}\text { Variable explicativa discreta. Indica el número de empresas asociadas que par- } \\
\text { ticiparon en el proyecto de } \mathrm{I}+\mathrm{D} \text { analizado. }\end{array}$ \\
\hline & $\begin{array}{l}\text { Número de Institu- } \\
\text { ciones Extranjeras } \\
\text { Asociadas }\end{array}$ & $\mathrm{n}^{\circ}$ extranjeras & $\begin{array}{l}\text { Variable explicativa discreta. Indica el número de instituciones extranjeras que } \\
\text { participaron en el proyecto de I+D analizado. }\end{array}$ \\
\hline & $\begin{array}{l}\text { Número de Orga- } \\
\text { nismos Públicos } \\
\text { Asociados }\end{array}$ & $\mathrm{n}^{\circ}$ org públicos & $\begin{array}{l}\text { Variable explicativa discreta. Indica el número de instituciones públicas que } \\
\text { participaron en el proyecto de } \mathrm{I}+\mathrm{D} \text { analizado. }\end{array}$ \\
\hline & $\begin{array}{l}\text { Número de Orga- } \\
\text { nizaciones Sociales } \\
\text { Asociadas }\end{array}$ & $\mathrm{n}^{\circ}$ org sociales & $\begin{array}{l}\text { Variable explicativa discreta. Indica el número de organizaciones sociales que } \\
\text { participaron en el proyecto de I+D analizado. }\end{array}$ \\
\hline $\begin{array}{l}\text { Estructura de Soporte Aca- } \\
\text { démica (explicativa) }\end{array}$ & Tamaño Facultad & tamaño facultad & $\begin{array}{l}\text { Variable explicativa discreta. Indica el tamaño de la facultad a la que pertenece } \\
\text { el director del proyecto de I+D analizado, medido por la cantidad académicos } \\
\text { contratados a jornada completa de } 44 \text { horas que posee la facultad. }\end{array}$ \\
\hline
\end{tabular}

Fuente: Elaboración Propia 


\section{Regresión Probit}

En los modelos de regresión lineal se tiene una o múltiples variables explicativas $(\mathrm{X})$ y una variable dependiente $(\mathrm{Y})$ de naturaleza aleatoria continua. En algunas situaciones, la variable dependiente puede ser categórica, por lo cual puede adoptar un número limitado de categorías, a las cuales se asignan determinados valores. En tal situación, el método de mínimos cuadrados no es apropiado para obtener buenas aproximaciones (Menezes, Liska, Cirillo, \& Vivanco, 2017). Una mejor estimación se obtiene por el modelo de regresión probit, ya que permite el uso de un modelo de regresión para calcular la probabilidad específica de ocurrencia de un evento definido como p(x) (Atkinson, 1985). Así, el modelo de regresión planteado se utiliza cuando se desea pronosticar la probabilidad de que ocurra o no un suceso determinado (Rojo, 2007), en donde los eventos toman valores de $1 \mathrm{o}$ 0 , en caso de ocurrir o no respectivamente.

En la presente investigación, la variable dependiente será del tipo categórica binaria o dicotómica, en donde se obedecerá los siguientes valores:

$Y($ dicotómica $)=\left\{\begin{array}{l}1 \rightarrow \text { Proyecto ha transferido tecnología } \\ 0 \rightarrow \text { Proyecto no ha transferido tecnología }\end{array}\right.$

El objetivo de aplicar la metodología probit fue determinar la relación existente entre las distintas variables de proyectos, definidas como explicativas y que afectan la transferencia tecnológica y el efectivo proceso de transferencia tecnológica de los proyectos, considerada como variable dependiente.

\section{Resultados}

Se realizaron estimaciones en base al modelo probit para evaluar la influencia de las variables explicativas en la variable dependiente, considerando ocho modelos en los cuales las variables fueron introducidas gradualmente.

Dado que los proyectos incluidos dentro de la muestra se desarrollaron durante distintos períodos de tiempo, resulta necesario evaluar si el año de término del proyecto influye de manera significativa en la determinación de la probabilidad de transferencia tecnológica. En este ámbito, se observa que existe una concentración, dentro de la muestra, de proyectos terminados en los últimos tres años. Así, el 63\% de los proyectos muestreados finalizó su ejecución entre los años 2005-2013, mientras que un 37\% lo hicieron dentro del período 2014-2016, mostrando un considerable aumento de los proyectos finalizados en la Universidad de Concepción en el último período. Dado lo anterior, resulta de interés evaluar los determinantes de la transferencia tecnológica en la universidad según el momento del tiempo en el cual se cerró el proyecto de $\mathrm{I}+\mathrm{D}$, con el fin de identificar si los determinantes de la transferencia tecnológica de los proyectos cambian según el período de tiempo en que se desarrollaron. Así, en primer lugar, se muestran los resultados obtenidos a partir del modelo aplicado a la muestra total de proyectos (ver Tablas 2 y 3 ). 
Tabla 2

Análisis estadístico 2005-2016

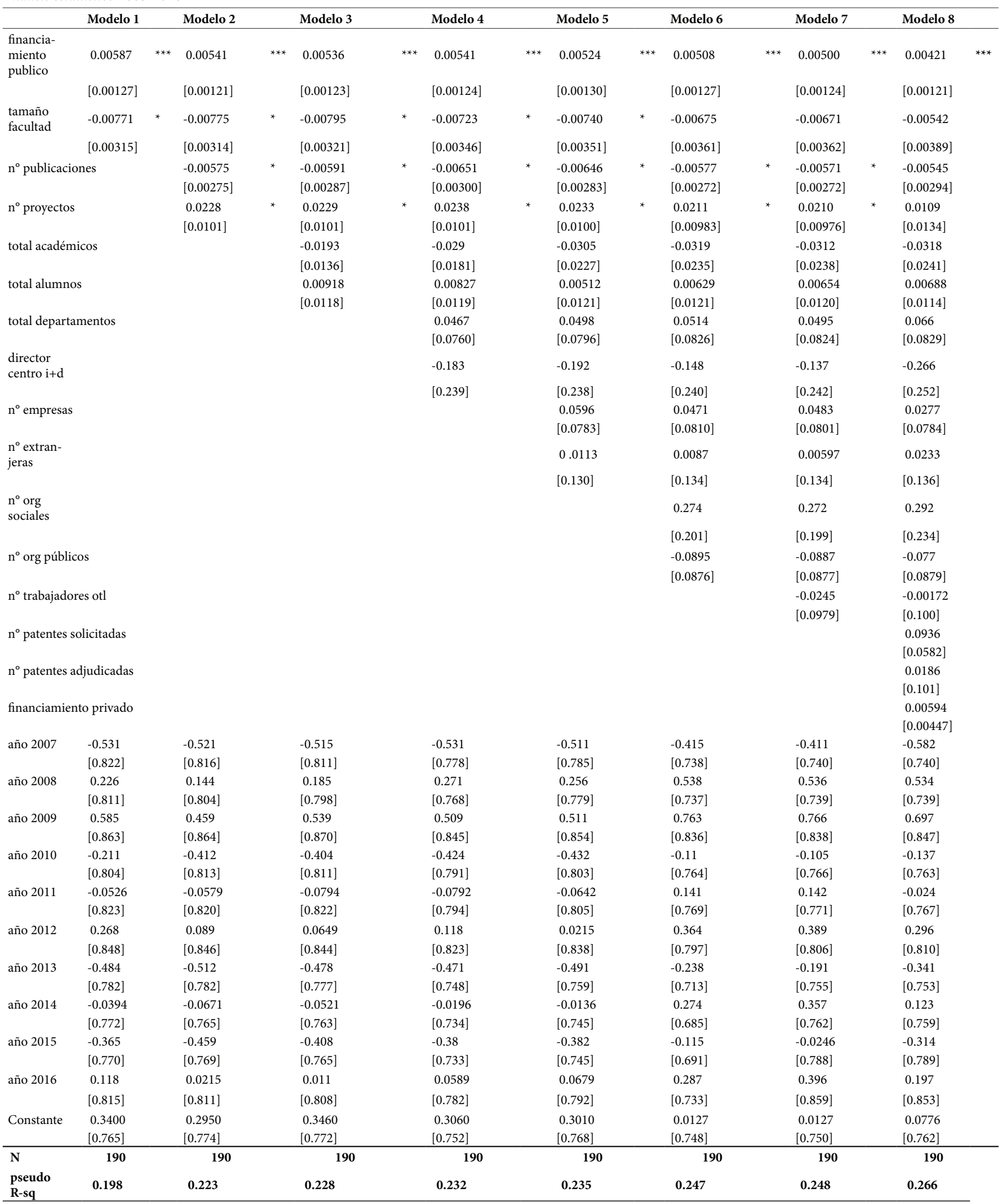

Fuente: Elaboración propia. Nota: ${ }^{\star} \mathrm{p}<0.05,{ }^{* *} \mathrm{p}<0.01,{ }^{* * *} \mathrm{p}<0.001$

ISSN: 0718-2724. (http://jotmi.org)

Journal of Technology Management \& Innovation (c) Universidad Alberto Hurtado, Facultad de Economía y Negocios. 
Tabla 3

Contribución marginal variables explicativas del 8vo modelo 2005-2016

\begin{tabular}{|c|c|c|c|c|c|c|c|}
\hline Variable & $\mathrm{dy} / \mathrm{dx}$ & Std. Err. & $\mathbf{z}$ & $\mathbf{P}>\mathbf{Z}$ & {$[95 \%$} & C.I. ] & $\mathbf{X}$ \\
\hline financiamiento publico & 0.0013722 & 0.00035 & 3.92 & 0.000 & 0.000685 & 0.002059 & 137.4240 \\
\hline tamaño facultad & -0.0017641 & 0.00131 & -1.34 & 0.179 & -0.004337 & 0.000808 & 620.8420 \\
\hline $\mathrm{n}^{\circ}$ publicaciones & -0.0017768 & 0.00094 & -1.88 & 0.060 & -0.003628 & 0.000075 & 43.70000 \\
\hline $\mathrm{n}^{\circ}$ proyectos & 0.0035447 & 0.00432 & 0.82 & 0.412 & -0.004919 & 0.012008 & 187.3680 \\
\hline total académicos & -0.0103640 & 0.00786 & -1.32 & 0.187 & -0.025774 & 0.005046 & 498.4210 \\
\hline total alumnos & 0.0022399 & 0.00374 & 0.60 & 0.550 & -0.005096 & 0.009576 & 585.2630 \\
\hline total departamentos & 0.0215001 & 0.02710 & 0.79 & 0.428 & -0.031620 & 0.074620 & 245.7890 \\
\hline director centro $\mathrm{i}+\mathrm{d}$ & -0.0869518 & 0.08240 & -1.06 & 0.291 & -0.248454 & 0.074551 & 0.473684 \\
\hline $\mathrm{n}^{\circ}$ empresas & 0.0090248 & 0.02576 & 0.35 & 0.726 & -0.041464 & 0.059513 & 104.2110 \\
\hline $\mathrm{n}^{\circ}$ extranjeras & 0.0076025 & 0.04418 & 0.17 & 0.863 & -0.078994 & 0.094199 & 0.689474 \\
\hline $\mathrm{n}^{\circ}$ org sociales & 0.0951771 & 0.07286 & 1.31 & 0.191 & -0.047621 & 0.237975 & 0.378947 \\
\hline $\mathrm{n}^{\circ}$ org publicas & -0.0250901 & 0.02875 & -0.87 & 0.383 & -0.081436 & 0.031256 & 0.636842 \\
\hline $\mathrm{n}^{\circ}$ trabajadores otl & -0.0005613 & 0.03270 & -0.02 & 0.986 & -0.064643 & 0.063520 & 172.6320 \\
\hline $\mathrm{n}^{\circ}$ patentes solicitadas & 0.0304772 & 0.01936 & 1.57 & 0.115 & -0.007475 & 0.068429 & 210.5260 \\
\hline $\mathrm{n}^{\circ}$ patentes adjudicadas & 0.0060682 & 0.03298 & 0.18 & 0.854 & -0.058572 & 0.070708 & 112.6320 \\
\hline financiamiento privado & 0.0019363 & 0.00140 & 1.39 & 0.165 & -0.000798 & 0.004671 & 210.0660 \\
\hline año2007 & -0.2126262 & 0.29106 & -0.73 & 0.465 & -0.783095 & 0.357843 & 0.078947 \\
\hline año2008 & 0.1474601 & 0.16342 & 0.90 & 0.367 & -0.172839 & 0.467760 & 0.094737 \\
\hline año2009 & 0.1775499 & 0.15103 & 1.18 & 0.240 & -0.118472 & 0.473572 & 0.057895 \\
\hline año2010 & -0.0462818 & 0.26593 & -0.17 & 0.862 & -0.567490 & 0.474926 & 0.089474 \\
\hline año2011 & -0.0078683 & 0.25327 & -0.03 & 0.975 & -0.504270 & 0.488533 & 0.084211 \\
\hline año2012 & 0.0883565 & 0.21562 & 0.41 & 0.682 & -0.334248 & 0.510961 & 0.078947 \\
\hline año2013 & -0.1188717 & 0.27917 & -0.43 & 0.670 & -0.666028 & 0.428285 & 0.131579 \\
\hline año2014 & 0.0388916 & 0.23204 & 0.17 & 0.867 & -0.415896 & 0.493679 & 0.136842 \\
\hline año2015 & -0.1083757 & 0.28689 & -0.38 & 0.706 & -0.670662 & 0.453911 & 0.168421 \\
\hline año2016 & 0.0604396 & 0.24502 & 0.25 & 0.805 & -0.419800 & 0.540679 & 0.068421 \\
\hline
\end{tabular}

Fuente: Elaboración propia.

A partir de las Tablas 2 y 3 , no se observa un efecto estadísticamente significativo en la determinación de la probabilidad de transferencia tecnológica por parte de alguna de las variables asociadas al año de término del proyecto. Al mismo tiempo, se observa que el financiamiento público de $\mathrm{I}+\mathrm{D}$ recibido por el proyecto, tiene un impacto positivo estadísticamente significativo en la probabilidad de transferencia tecnológica en todos los modelos evaluados. Lo anterior indica que, para cada año muestreado, el financiamiento que recibe el proyecto por parte del sector público determina en gran medida el éxito del proyecto en transferir tecnología. Por otro lado, se observa que el número de publicaciones que posee el director del proyecto, muestra un efecto negativo estadísticamente significativo sobre la probabilidad de transferencia tecnológica, mientras que, el número de proyectos de $\mathrm{I}+\mathrm{D}$ en el cual ha participado el investigador tiene un impacto positivo estadísticamente significativo sobre dicha probabilidad en seis de ocho modelos evaluados.
Adicionalmente, se evaluaron los determinantes de la transferencia tecnológica separando la muestra obtenida en dos períodos. El primero, en base al período 2005-2013, comprende un lapsus de nueve años, en el cual se cerraron 119 proyectos de $\mathrm{I}+\mathrm{D}$ pertenecientes a la muestra. El segundo, basado en el periodo 2014-2016, abarca un total de tres años, en el cual se cerraron 71 proyectos de I+D pertenecientes a la muestra. Se puede observar, que la cantidad de proyectos cerrados en la muestra aumenta de manera considerable dentro del último período, por lo cual la separación de los periodos permite obtener antecedentes actualizados acerca de los factores que explican la variable dependiente, como también identificar si han existido cambios en los determinantes de la transferencia tecnológica en la Universidad de Concepción dentro de los últimos años. En Tablas 4 y 5 se presentan los resultados obtenidos para los determinantes de la transferencia tecnológica. En relación al período comprendido entre los años 2005 y 2013, en Tablas 4 y 5 se presentan los resultados obtenidos para los determinantes de la transferencia tecnológica. 
Tabla 4

Análisis estadístico 2005-2013

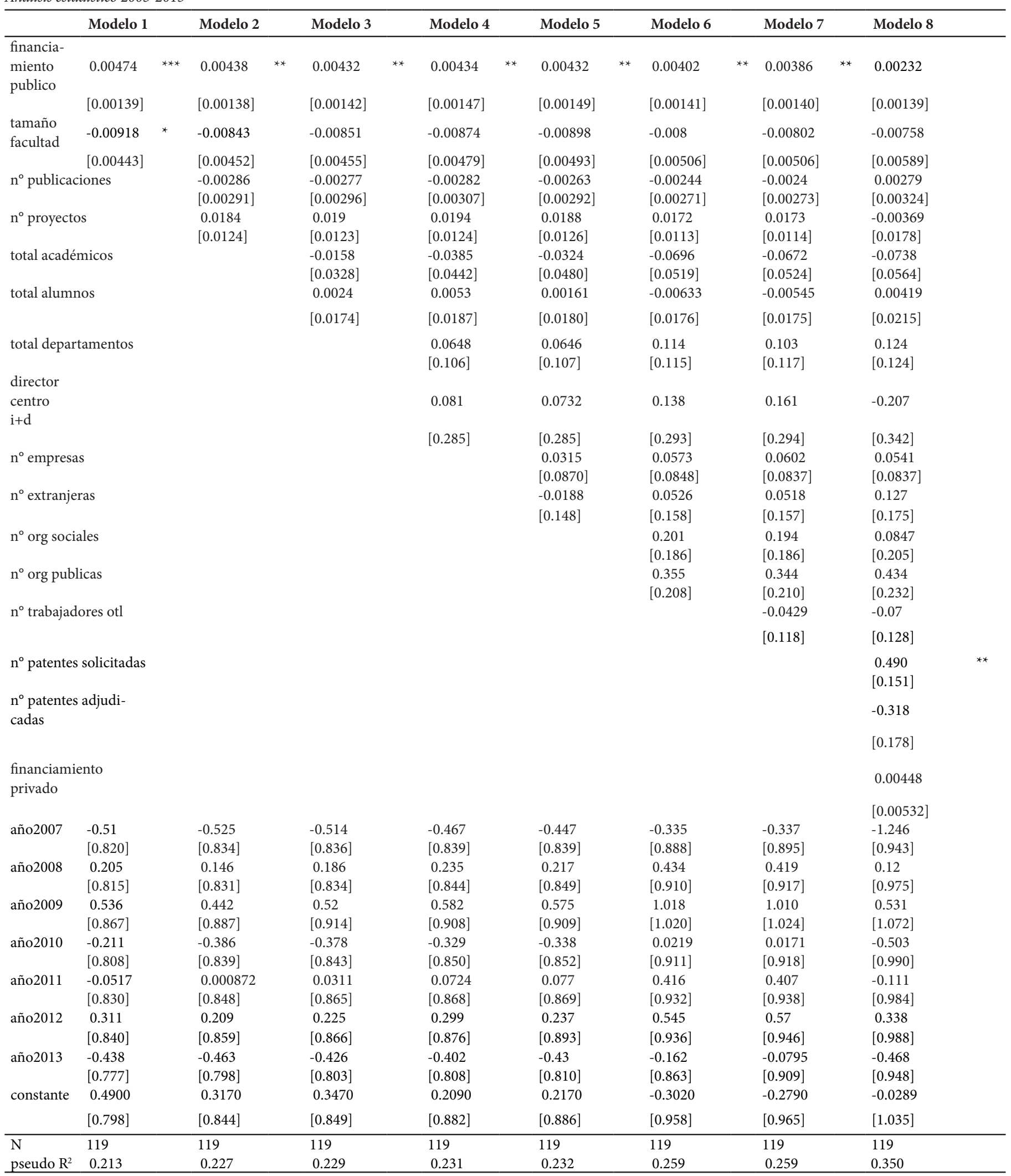

Fuente: Elaboración propia. Nota: ${ }^{*} \mathrm{p}<0.05,{ }^{*} \mathrm{p}<0.01,{ }^{* *} \mathrm{p}<0.001$. 
Tabla 5

Contribución marginal variables explicativas del 8vo modelo 2005-2013

\begin{tabular}{|c|c|c|c|c|c|c|c|}
\hline Variable & $\mathrm{dy} / \mathrm{dx}$ & Std. Err. & $\mathbf{z}$ & $\mathbf{P}>\mathbf{z}$ & [ $95 \%$ & C.I. ] & $\mathbf{X}$ \\
\hline $\mathrm{n}^{\circ}$ publicaciones & 0.0007681 & 0.00091 & 0.84 & 0.400 & -0.001021 & 0.002558 & 521.7650 \\
\hline $\mathrm{n}^{\circ}$ proyectos & -0.0010177 & 0.00495 & -0.21 & 0.837 & -0.010718 & 0.008682 & 21.04200 \\
\hline total alumnos & 0.0011552 & 0.00595 & 0.19 & 0.846 & -0.010511 & 0.012822 & 571.4290 \\
\hline total departamentos & 0.0341966 & 0.03461 & 0.99 & 0.323 & -0.033646 & 0.102040 & 258.8240 \\
\hline director centro $\mathrm{i}+\mathrm{d}$ & -0.0576184 & 0.09634 & -0.60 & 0.550 & -0.246450 & 0.131213 & 0.436975 \\
\hline $\mathrm{n}^{\circ}$ org sociales & 0.0233596 & 0.05631 & 0.41 & 0.678 & -0.087013 & 0.133732 & 0.478992 \\
\hline $\mathrm{n}^{\circ}$ org publicas & 0.1196220 & 0.06320 & 1.89 & 0.058 & -0.004253 & 0.243497 & 0.579832 \\
\hline $\mathrm{n}^{\circ}$ trabajadores otl & -0.0192898 & 0.03561 & -0.54 & 0.588 & -0.089075 & 0.050496 & 0.537815 \\
\hline $\mathrm{n}^{\circ}$ patentes solicitadas & 0.1350896 & 0.04216 & 3.20 & 0.001 & 0.052454 & 0.217725 & 210.0840 \\
\hline $\mathrm{n}^{\circ}$ patentes adjudicadas & -0.0875361 & 0.04591 & -1.91 & 0.057 & -0.177525 & 0.002453 & 112.6050 \\
\hline financiamiento privado & 0.0012346 & 0.00139 & 0.89 & 0.373 & -0.001482 & 0.003952 & 270.2920 \\
\hline año2007 & -0.4358726 & 0.35041 & -1.24 & 0.214 & -1.122670 & 0.250921 & 0.126050 \\
\hline año2012 & 0.0829778 & 0.21264 & 0.39 & 0.696 & -0.333782 & 0.499738 & 0.126050 \\
\hline año2013 & -0.1431503 & 0.31616 & -0.45 & 0.651 & -0.762818 & 0.476517 & 0.210084 \\
\hline
\end{tabular}

Fuente: Elaboración propia.

A partir de las Tablas 4 y 5 se observa que el financiamiento público de I+D impacta de forma positiva y estadísticamente significativa en siete de los ocho modelos evaluados en el periodo 2005-2013. Lo anterior indica que el aporte financiero recibido por parte del sector público se mantiene como un factor que incide en la determinación de la probabilidad de transferencia. Sin embargo, pierde significancia estadística como variable explicativa dentro del último modelo evaluado. Al mismo tiempo, se observa que su contribución marginal a la probabilidad de transferencia tecnológica disminuye en relación al modelo global condicionado por año, dejando de ser estadísticamen- te significativa. Por otro lado, se obtiene que el número de patentes solicitadas que posee el director del proyecto impacta de forma positiva y estadísticamente significativa la probabilidad de transferencia tecnológica. En cuanto a su contribución marginal, se obtiene que por cada solicitud de patente extra que posee el director de proyecto la probabilidad de transferir tecnología aumenta en un 13,5\%.

En relación al segundo período, entre 2014 y 2016, los resultados obtenidos para los determinantes de la transferencia tecnológica se ilustran en Tablas 6 y 7. 
Tabla 6

Análisis estadístico 2014-2016

\begin{tabular}{|c|c|c|c|c|c|c|c|c|c|c|c|c|c|c|c|c|}
\hline & Modelo 1 & & Modelo 2 & & Modelo 3 & & Modelo 4 & & Modelo 5 & & Modelo 6 & & Modelo 7 & & Modelo 8 & \\
\hline \multirow[t]{2}{*}{ financiamiento publico } & 0.00915 & $* * *$ & 0.00814 & $* *$ & 0.00860 & ** & 0.00858 & ** & 0.00659 & * & 0.00692 & ** & 0.00765 & $* *$ & 0.00909 & $* *$ \\
\hline & {$[0.00272]$} & & {$[0.00255]$} & & {$[0.00263]$} & & {$[0.00277]$} & & {$[0.00292]$} & & {$[0.00251]$} & & {$[0.00272]$} & & {$[0.00289]$} & \\
\hline \multirow[t]{2}{*}{ tamaño facultad } & -0.00702 & & -0.00717 & & -0.00729 & & -0.00431 & & -0.00196 & & -0.00367 & & -0.00415 & & -0.000683 & \\
\hline & {$[0.00478]$} & & {$[0.00466]$} & & {$[0.00476]$} & & {$[0.00538]$} & & {$[0.00565]$} & & {$[0.00664]$} & & {$[0.00729]$} & & {$[0.00805]$} & \\
\hline \multirow[t]{2}{*}{$\mathrm{n}^{\circ}$ publicaciones } & & & -0.0142 & $\star *$ & -0.0162 & $* *$ & -0.0189 & $\star *$ & $-0.0182^{*}$ & & -0.0189 & * & -0.0261 & * & -0.0253 & $* *$ \\
\hline & & & {$[0.00508]$} & & {$[0.00521]$} & & {$[0.00732]$} & & {$[0.00750]$} & & {$[0.00929]$} & & {$[0.0122]$} & & {$[0.00932]$} & \\
\hline \multirow[t]{2}{*}{$\mathrm{n}^{\circ}$ proyectos } & & & 0.0232 & & 0.0200 & & 0.0378 & & 0.0383 & & 0.042 & & 0.0584 & & 0.0278 & \\
\hline & & & {$[0.0172]$} & & {$[0.0167]$} & & {$[0.0233]$} & & {$[0.0214]$} & & {$[0.0283]$} & & {$[0.0330]$} & & {$[0.0328]$} & \\
\hline \multirow[t]{2}{*}{ total académicos } & & & & & -0.0323 & * & -0.0397 & & -0.0448 & & -0.02 & & -0.0548 & & -0.0341 & \\
\hline & & & & & {$[0.0144]$} & & {$[0.0227]$} & & {$[0.0387]$} & & {$[0.0519]$} & & {$[0.0599]$} & & {$[0.0615]$} & \\
\hline \multirow[t]{2}{*}{ total alumnos } & & & & & 0.0235 & & 0.0173 & & 0.0166 & & 0.0252 & & 0.0234 & & 0.0208 & \\
\hline & & & & & {$[0.0133]$} & & {$[0.0132]$} & & {$[0.0136]$} & & {$[0.0137]$} & & {$[0.0140]$} & & {$[0.0144]$} & \\
\hline \multirow[t]{2}{*}{ total departamentos } & & & & & & & 0.0641 & & 0.0571 & & 0.104 & & 0.131 & & 0.125 & \\
\hline & & & & & & & {$[0.134]$} & & {$[0.144]$} & & {$[0.134]$} & & {$[0.145]$} & & {$[0.157]$} & \\
\hline \multirow[t]{2}{*}{ director centro $\mathrm{i}+\mathrm{d}$} & & & & & & & -0.684 & & -0.761 & & -0.732 & & -0.971 & & -1.034 & \\
\hline & & & & & & & {$[0.421]$} & & {$[0.414]$} & & {$[0.469]$} & & {$[0.539]$} & & {$[0.574]$} & \\
\hline \multirow[t]{2}{*}{$\mathrm{n}^{\circ}$ empresas } & & & & & & & & & 0.247 & & 0.162 & & 0.154 & & -0.14 & \\
\hline & & & & & & & & & {$[0.188]$} & & {$[0.196]$} & & {$[0.205]$} & & {$[0.201]$} & \\
\hline \multirow[t]{2}{*}{$\mathrm{n}^{\circ}$ extranjeras } & & & & & & & & & 0.0247 & & -0.497 & & -0.295 & & -0.463 & \\
\hline & & & & & & & & & {$[0.291]$} & & {$[0.408]$} & & {$[0.421]$} & & {$[0.461]$} & \\
\hline \multirow[t]{2}{*}{$n^{\circ}$ org sociales } & & & & & & & & & & & 1.849 & ** & 1.967 & $* *$ & 2.437 & $* *$ \\
\hline & & & & & & & & & & & {$[0.654]$} & & {$[0.755]$} & & {$[0.813]$} & \\
\hline \multirow[t]{2}{*}{$\mathrm{n}^{\circ}$ org publicas } & & & & & & & & & & & $-0.772^{*}$ & & -0.973 & $*$ & -0.862 & * \\
\hline & & & & & & & & & & & {$[0.381]$} & & {$[0.463]$} & & {$[0.436]$} & \\
\hline \multirow[t]{2}{*}{$\mathrm{n}^{\circ}$ trabajadores otl } & & & & & & & & & & & & & 0.271 & & 0.367 & \\
\hline & & & & & & & & & & & & & {$[0.226]$} & & {$[0.228]$} & \\
\hline \multirow[t]{2}{*}{$\mathrm{n}^{\circ}$ patentes solicitadas } & & & & & & & & & & & & & & & 0.181 & \\
\hline & & & & & & & & & & & & & & & {$[0.103]$} & \\
\hline \multirow[t]{2}{*}{$\mathrm{n}^{\circ}$ patentes adjudicadas } & & & & & & & & & & & & & & & 0.100 & \\
\hline & & & & & & & & & & & & & & & {$[0.164]$} & \\
\hline \multirow[t]{2}{*}{ financiamiento privado } & & & & & & & & & & & & & & & 0.0186 & * \\
\hline & & & & & & & & & & & & & & & {$[0.00795]$} & \\
\hline \multirow[t]{2}{*}{ año2014 } & -0.213 & & -0.0981 & & -0.0787 & & -0.186 & & -0.17 & & 0.184 & & 0.62 & & 0.562 & \\
\hline & {$[0.465]$} & & {$[0.460]$} & & {$[0.472]$} & & {$[0.515]$} & & {$[0.505]$} & & [0.587] & & {$[0.678]$} & & {$[0.686]$} & \\
\hline \multirow[t]{2}{*}{ año2015 } & -0.633 & & -0.533 & & -0.446 & & -0.549 & & -0.525 & & -0.506 & & -0.238 & & -0.162 & \\
\hline & [0.493] & & {$[0.512]$} & & {$[0.522]$} & & {$[0.524]$} & & {$[0.521]$} & & [0.597] & & {$[0.635]$} & & {$[0.635]$} & \\
\hline \multirow[t]{2}{*}{ constante } & 0.275 & & 0.342 & & 0.431 & & 0.399 & & 0.205 & & 0.364 & & -0.714 & & -1.333 & \\
\hline & {$[0.546]$} & & {$[0.554]$} & & {$[0.566]$} & & {$[0.613]$} & & {$[0.653]$} & & {$[0.873]$} & & [1.141] & & [1.295] & \\
\hline $\mathrm{N}$ & 71 & & 71 & & 71 & & 71 & & 71 & & 71 & & 71 & & 71 & \\
\hline pseudo $\mathrm{R}^{2}$ & 0.187 & & 0.245 & & 0.274 & & 0.301 & & 0.320 & & 0.422 & & 0.439 & & 0.469 & \\
\hline
\end{tabular}

Fuente: Elaboración propia. Nota: ${ }^{\star} \mathrm{p}<0.05,{ }^{* *} \mathrm{p}<0.01,{ }^{* * *} \mathrm{p}<0.001$ 
Tabla 7

Contribución marginal variables explicativas del 8vo modelo 2014-2016

\begin{tabular}{|c|c|c|c|c|c|c|c|}
\hline Variable & $d y / d x$ & Std. Err. & $\mathbf{z}$ & $\mathbf{P}>\mathbf{z}$ & [ $95 \%$ & C.I. ] & $\mathbf{X}$ \\
\hline financiamiento publico & 0.0030111 & 0.00088 & 3.44 & 0.001 & 0.001296 & 0.004726 & 102.2600 \\
\hline $\mathrm{n}^{\circ}$ publicaciones & -0.0083726 & 0.00279 & -3.00 & 0.003 & -0.013841 & -0.002904 & 29.49300 \\
\hline total académicos & -0.0112897 & 0.02009 & -0.56 & 0.574 & -0.050658 & 0.028079 & 508.4510 \\
\hline total alumnos & 0.0068833 & 0.00498 & 1.38 & 0.167 & -0.002882 & 0.016649 & 608.4510 \\
\hline total departamentos & 0.0413908 & 0.05190 & 0.80 & 0.425 & -0.060322 & 0.143104 & 223.9440 \\
\hline director centro $\mathrm{i}+\mathrm{d}$ & -0.3262934 & 0.16856 & -1.94 & 0.053 & -0.656661 & 0.004074 & 0.535211 \\
\hline $\mathrm{n}^{\circ}$ org sociales & 0.8071220 & 0.27041 & 2.98 & 0.003 & 0.277136 & 133.7110 & 0.211268 \\
\hline $\mathrm{n}^{\circ}$ org publicas & -0.2856554 & 0.15963 & -1.79 & 0.074 & -0.598528 & 0.027217 & 0.732394 \\
\hline $\mathrm{n}^{\circ}$ trabajadores otl & 0.1215766 & 0.07448 & 1.63 & 0.103 & -0.024401 & 0.267554 & 371.8310 \\
\hline $\mathrm{n}^{\circ}$ patentes solicitadas & 0.0600045 & 0.03298 & 1.82 & 0.069 & -0.004629 & 0.124638 & 211.2680 \\
\hline $\mathrm{n}^{\circ}$ patentes adjudicadas & 0.0332367 & 0.05366 & 0.62 & 0.536 & -0.071927 & 0.138400 & 112.6760 \\
\hline financiamiento privado & 0.0061668 & 0.00262 & 2.35 & 0.019 & 0.001023 & 0.011310 & 109.1230 \\
\hline año2014 & 0.1760020 & 0.20195 & 0.87 & 0.383 & -0.219816 & 0.571820 & 0.366197 \\
\hline
\end{tabular}

Fuente: Elaboración propia.

De las Tablas 6 y 7 se obtiene que el financiamiento público de $\mathrm{I}+\mathrm{D}$ recibido por el proyecto sigue siendo una variable que impacta de forma positiva y estadísticamente significativa la probabilidad de transferencia tecnológica en la Universidad de Concepción en el periodo 2014-2016. Se observa que la contribución marginal de la variable sobre la probabilidad de transferencia tecnológica, aumenta en comparación al modelo global condicionado por años. También se obtiene que el número de publicaciones que posee el director del proyecto de $\mathrm{I}+\mathrm{D}$, tiene un impacto negativo estadísticamente significativo sobre la probabilidad de transferencia tecnológica, dentro de todos los modelos estimados. La contribución marginal del número de publicaciones aumentó, indicando que, dentro del último período, la probabilidad de transferir tecnología disminuye un $0,84 \%$ por cada publicación científica extra que posee el director del proyecto de I+D. Por otro lado, se observa que otras variables presentan significancia estadística en la determinación de la transferencia tecnológica, entre ellas, el monto de financiamiento privado recibido por el proyecto de $\mathrm{I}+\mathrm{D}$ ha tomado relevancia en la determinación de la transferencia tecnológica dentro de los últimos tres años, mostrando un impacto positivo y estadísticamente significativo dentro del último modelo estimado. La contribución marginal del financiamiento recibido por parte de empresas indica que por cada millón de pesos que recibe el proyecto por parte del sector privado, la probabilidad de transferir tecnología aumenta en $0,62 \%$. También es posible observar que el número de organizaciones sociales que participa dentro del proyecto de $\mathrm{I}+\mathrm{D}$ ha tenido un impacto positivo y estadísticamente significativo en la transferencia tecnológica, dentro de todos los modelos evaluados. La contribución marginal de esta variable indica que por cada organización social que participa dentro del proyecto, la probabilidad de transferir tecnología aumenta en un 80,7\%. Finalmente, se observa que el número de organizaciones públicas que participan en el proyecto de $\mathrm{I}+\mathrm{D}$, determina de forma negativa y estadísticamente significativa la probabilidad de transferencia tecnológica. Su contribución marginal indica que por cada organismo público que participa en el proyecto, la probabilidad de transferir tecnología disminuye en $28,6 \%$.

\section{Discusión}

El financiamiento público de I+D ha sido un determinante importante para la transferencia tecnológica de la Universidad de Concepción a lo largo de los años, cobrando una papel aún más importante en el último período. Tal financiamiento permite generar nuevas 
instancias de desarrollo que pudieran tener un impacto significativo en el mercado. La investigación generada en las universidades tiende a ser embrionaria, por lo que es necesario que los resultados obtenidos sean validados técnica y comercialmente en diversas etapas y escalas lo que requiere una gran inversión. Dentro de la literatura, Foltz et al., (2000) y Azagra (2001) afirman que el financiamiento público es un factor importante para fomentar la transferencia de tecnologías desarrolladas en universidades, teniendo un impacto positivo y significativo en cuanto a la transferencia de conocimiento.

En relación a los resultados obtenidos para el número de publicaciones del director del proyecto, se observa que cumplen un papel importante en la determinación de la probabilidad de transferencia tecnológica en la Universidad de Concepción, cobrando un efecto negativo estadísticamente significativo dentro de los últimos tres años. Las publicaciones científicas generalmente se asocian a fines de investigación básica. Las capacidades de los investigadores dentro de la universidad se miden principalmente por lo académico, teniendo a las publicaciones como principal indicador de productividad científi$\mathrm{ca}$, mientras que su desempeño en temas de transferencia tecnológica no se considera de igual forma. Al mismo tiempo, las evaluaciones de las actividades de los grupos de investigación se respaldan en indicadores que se basan en medidas bibliométricas de volumen (número de publicaciones) y calidad (número de citas o factor de impacto), por lo cual las publicaciones científicas son altamente valoradas ${ }^{2}$. En este ámbito, la gradual mejora presentada por Chile en cuanto a volumen y calidad de sus publicaciones, no se condice aún con una mayor transferencia de tecnologías desarrolladas en el país hacia el sector productivo nacional.

Por otro lado, se observa que, en los últimos tres años, el financiamiento privado recibido por los proyectos ha cobrado significancia en la determinación de la transferencia tecnológica. El financiamiento privado que reciben los proyectos de la universidad por parte de empresas es bajo, debido a que el sector privado en Chile realiza poca inversión en $\mathrm{I}+\mathrm{D}$ por el riesgo que esto conlleva. A pesar de lo anterior, los resultados evidencian que, en los últimos años, tal financiamiento se ha vuelto un determinante de la probabilidad de transferencia tecnológica en la Universidad de Concepción. Al igual que el financiamiento público, el financiamiento privado aporta al nivel de inversión requerido para la validación técnica y comercial de las invenciones universitarias. Por otro lado, la cooperación entre la academia y la industria resulta ser clave para que las invenciones desarrolladas a nivel universitario tengan una orientación directa a los problemas y demandas del sector privado. Fernández et al., (2009), asegura que el financiamiento privado ha estado siempre más orientado a la obtención de resultados que puedan comercializarse en el corto y mediano plazo, contribuyendo a la generación de procesos de transferencia tecnológica efectivos.

Dentro del último período, también se observa una influencia relevante en la probabilidad de transferencia tecnológica por parte del número de organismos públicos que participan en el proyecto, afectando de forma negativa la probabilidad de transferir tecnología. En Chile, varios de los programas públicos que financian investigación universitaria buscan obtener resultados de aplicación, innovación, emprendimiento y comercialización. Sin embargo, es probable que los resultados obtenidos dentro del período se encuentren condicionados por el número de proyectos muestreados pertenecientes a la Facultad de Educación de la universidad, los que se realizaron en colaboración con diversos organismos públicos y asociados chilenos del área. En general, el concepto de transferencia tecnológica no se relaciona de manera adecuada con el ámbito de la educación, debido a que la principal finalidad al apoyar proyectos en el área de la educación se relaciona con innovar en metodologías de enseñanza o procesos que permitan tener un impacto en la sociedad, lo cual no está incluido dentro de las metodologías de transferencia tecnológica definidas en la presente investigación. El interés de las organizaciones públicas por respaldar proyectos de $\mathrm{I}+\mathrm{D}$ se basa en los potenciales beneficios de desarrollar resultados de la investigación científica que sirvan de respaldo al mercado y a la sociedad ${ }^{3}$.

También se observa en los últimos años, un efecto positivo y significativo del número de organizaciones sociales que participan dentro del proyecto. Las organizaciones sociales caracterizan a una sociedad en un determinado momento del tiempo. Dentro de los modelos de transferencia tecnológica universitaria, Carayannis \& Campbell (2012) destacan la importancia de la incorporación de la sociedad a los procesos de transferencia tecnológica, indicando que las personas y la sociedad aportan al proceso en ámbitos tan importantes como la cultura y la aceptación de las innovaciones. La sociedad es la encargada de legitimar las innovaciones generadas, por lo cual un buen respaldo de organizaciones sociales al proyecto de $\mathrm{I}+\mathrm{D}$, permite obtener resultados validados por un entorno social relacionado directamente con la invención.

Adicionalmente se obtuvo un efecto positivo estadísticamente significativo asociado al número de patentes solicitadas por el director del proyecto de I+D, dentro del período evaluado entre 2005 y 2013 . El número de patentes solicitadas se asocia a procesos de transferencia de conocimiento iniciados en el pasado, por lo cual actúa como indicador de las capacidades de investigación y experiencia del propio director de proyecto en el ámbito de transferencia tecnológica. En el mismo contexto, en relación al número de proyectos en los que ha participado el investigador previo a la realización del proyecto muestreado, se obtuvo un impacto positivo estadísticamente significativo en seis de ocho modelos considerando el total de la muestra, lo cual advierte la existencia de una relación con la variable dependiente que resulta interesante de explorar. Así, el número de proyectos y el número de patentes previas se asocian directamente con la experiencia del investigador, su trabajo previo y dedicación al área de la investigación. En la literatura, Kochenkova, Grimaldi \& Munari (2016), destaca la importancia que poseen los ámbitos de comunicación y de conocimiento en el proceso de transferencia tecnológica, indicando

${ }^{2}$ Estudio cualitativo sobre el estado actual de la transferencia tecnológica en Chile. Informe Final. Ministerio de Economía, Fomento y Turismo, 2016.
${ }^{3}$ Estudio cualitativo sobre el estado actual de la transferencia tecnológica en Chile. Informe Final. Ministerio de Economía, Fomento y Turismo, 2016. 
que, para poder transferir los resultados de proyectos realizados, se requiere tener experiencia en trabajo con empresas, con académicos de diversas áreas y con estudiantes, lo cual se refuerza con el trabajo reiterado, aumentando la confianza y la credibilidad del mismo investigador.

\section{Conclusiones}

La presente investigación analizó los determinantes de transferencia tecnológica en universidades chilenas, específicamente el caso de la Universidad de Concepción. Para esto, se utilizó una base de datos construida a partir de la recolección de antecedentes de 190 proyectos de $\mathrm{I}+\mathrm{D}$ que ha desarrollado la Universidad de Concepción desde el año 2005 hasta el año 2016.

Para poder dar respuesta a la pregunta de investigación planteada, se realizó un análisis cuantitativo a través de un modelo de regresión probit. Con esto se analizó la relación existente entre el proceso de transferencia tecnológica y las variables que afectan este proceso, las que se definieron en cinco conjuntos: Financiamiento de I+D, Capacidad de Investigación, Estructuras de Soporte Tecnológico, Redes y Estructura de Soporte Académica. En primer lugar, se calcularon los determinantes de transferencia tecnológica en base a un modelo global con el total de la muestra recopilada de proyectos de $\mathrm{I}+\mathrm{D}$, para posteriormente separar el modelo en dos sub-períodos condicionados por el año de término del proyecto, el primero entre 2005-2013 y el segundo entre 2014-2016, con el fin de identificar si los determinantes de la transferencia tecnológica en la Universidad de Concepción se ven condicionados por la localización temporal del proyecto. Los resultados del modelo global indicaron que el financiamiento público de $\mathrm{I}+\mathrm{D}$ incrementa la probabilidad que un proyecto de $\mathrm{I}+\mathrm{D}$ de la universidad pueda generar procesos de transferencia tecnológica a través de consultorías, contratos de $\mathrm{I}+\mathrm{D}$, licenciamientos, creación de spin-offs o generación de proceso de patentamiento. En relación al período 2005-2013, se obtuvo como determinantes de la transferencia tecnológica el financiamiento público de $\mathrm{I}+\mathrm{D}$ y el número de patentes solicitadas, ambas variables aumentando la probabilidad de transferencia tecnológica. Para el período 2014 a 2016, se identifican como determinantes de la transferencia tecnológica el financiamiento público de $\mathrm{I}+\mathrm{D}$, el financiamiento privado de $\mathrm{I}+\mathrm{D}$, el número de organizaciones sociales, el número de organismos públicos y el número de publicaciones del investigador. Las tres primeras variables aumentan la probabilidad de transferencia tecnológica, mientras que las últimas dos variables impactan negativamente la variable estudiada. Así, se considera que es la variable más adecuada para explicar, de forma transversal en el tiempo, la probabilidad de transferencia tecnológica en la Universidad de Concepción.

Existen alternativas diversas para acceder a recursos públicos que cofinancian los gastos en tecnología e innovación. Sin embargo, no solo la falta de recursos públicos limita el crecimiento del gasto en I+D por parte del sector privado chileno. Existen barreras adicionales, propias de ámbitos regulatorios y administrativos, en las cuales el Estado cumple un rol fundamental. Para superar tales limitaciones, desde el sector público se debiese perseverar e intensificar las actuales políticas destinadas a fomentar la competencia, incentivar la creación de empresas y reducir las barreras administrativas existentes al día de hoy. El Estado también debe fomentar en mayor medida las políticas de incentivos tributarios existentes para incentivar la instalación de grandes empresas tecnológicas internacionales en el país. Por otro lado, el networking y la creación de redes también requieren un impulso por parte del sector público, generando conexiones entre mentores, mercados e inversionistas en el área de la ciencia, tecnología e innovación. Así, se sugiere generar mayores y mejores condiciones para fortalecer la I+D en el sector privado, facilitar la iniciación y cierre de empresas, desburocratizar y eliminar regulaciones excesivas, cambiar la percepción negativa del fracaso en emprendimientos previos y eliminar las barreras de entrada en los mercados, lo cual va de la mano con el fortalecimiento de la competencia y la generación de la necesidad de innovar y mejorar la tecnología existente para mantenerse competitivo dentro del mercado. Adicionalmente, se debiese explorar incrementar los subsidios a la I+D y los fondos destinados al fortalecimiento de la base científico-tecnológica del país, la subvención de procesos de comercialización, subsidios para apoyar la creación y crecimiento de empresas innovadoras en etapas tempranas y, aumentar el financiamiento para establecer oficinas de transferencia tecnológica, incubadoras de empresas tecnológicas y parques científicos.

Las barreras administrativas propias del Estado, pueden tener estrecha relación con el impacto negativo obtenido por parte del número de organizaciones públicas sobre la transferencia tecnológica en la Universidad de Concepción. Así, los organismos públicos que se involucran en áreas de investigación universitaria pudiesen flexibilizar sus requisitos exigidos a los proyectos de $\mathrm{I}+\mathrm{D}$ que apoyan, centrando su aporte en un impacto positivo para la industria en colaboración con la sociedad.

Por otro lado, el número de proyectos en los cuales ha participado el investigador que desarrolla un proyecto de $\mathrm{I}+\mathrm{D}$ dentro de la Universidad de Concepción resultó ser determinante para la probabilidad de transferir tecnología al mercado dentro de seis de ocho modelos evaluados en el periodo 2005-2016. De manera similar, las solicitudes de patente del investigador principal del proyecto resultaron ser determinantes en la transferencia tecnológica durante el período 20052013. Ambas variables se relacionan directamente con la experiencia que posee el investigador en el ámbito de participación en proyectos de $\mathrm{I}+\mathrm{D}$ e iniciación de procesos de transferencia tecnológica. Dentro de cualquier universidad, la participación en proyectos de I+D y la realización de investigaciones que generen procesos de patentamiento, le permite al investigador desarrollar una experiencia valiosa en el ámbito de la transferencia tecnológica. Kochenkova et al., (2016) destaca, entre las principales ineficiencias que impiden alcanzar el óptimo social en materia de transferencia e innovación tecnológica, la falta de comunicación y de conocimiento. En general, existe una brecha de conocimiento entre los investigadores, emprendedores y el personal de las OTLs, lo cual afecta las posibilidades de transferencia tecnológica de los proyectos asociados. Mientras que algunos investigadores poseen habilidades y experiencia que aumentan sus posibilidades de transferencia tecnológica, otros carecen de ellas, 
generando disminución de estas probabilidades. Por lo anterior, se deben potenciar programas internos a las instituciones y políticas públicas a nivel nacional, que creen programas de entrenamiento y desarrollo de competencias para la transferencia tecnológica y comercialización de tecnologías.

En cuanto a las publicaciones científicas, resultaron afectar de manera negativa la probabilidad de transferencia tecnológica en la Universidad de Concepción en el último período evaluado. Dentro del ámbito académico, las publicaciones científicas, tanto ISI como SCIELO, tienden a ser relacionadas con estudios de investigación básica. Al mismo tiempo, las capacidades de investigación de los propios académicos universitarios se miden por criterios de productividad científi$\mathrm{ca}$, más que por sus capacidades de transferencia tecnológica, dando la impresión de que las publicaciones académicas son excesivamente valoradas dentro del prestigio académico. Dentro de la Universidad de Concepción, el enfoque de investigación básica de los académicos pudiese guiar sus proyectos hacia un entorno de menor aplicación e interacción con la industria, disminuyendo las probabilidades de transferencia tecnológica. Así, el desarrollo de más investigación aplicada con impacto en la sociedad resulta clave para vincular el mundo universitario con las necesidades de las empresas y sectores productivos. Para esto, el sector público cumple un rol importante promoviendo mediante financiamiento de $\mathrm{I}+\mathrm{D}$ la investigación aplicada. Lo anterior, no necesariamente va en deterioro de la investigación científica básica, pero si amerita una mirada particular desde el punto de vista de la formación académica, de la asignación de recursos públicos y el cofinanciamiento del sector privado.

Los factores identificados en el presente trabajo como determinantes de la transferencia tecnológica en la Universidad de Concepción, pueden constituir nuevo conocimiento útil para la formulación de protocolos institucionales y políticas públicas por parte de hacedores de política y autoridades académicas, pudiendo resultar ello en una mejora de la gestión de la ciencia, tecnología e innovación.

\section{Referencias}

Algieri, B., Aquino, A., \& Succurro, M. (2013). Technology transfer offices and academic spin-off creation: the case of Italy. The Journal of Technology Transfer, 38(4), 382-400. doi:10.1007/s10961-011-9241-8

Atkinson, A. C. (1985). Plots, Transformations and Regression: An Introduction to Graphical Methods of Diagnostic Regression Analysis. Technical Report. Oxford: Oxford University Press.

Azagra, J. M. (2001). Determinantes de las patentes universitarias: el caso de la Universidad Politécnica de Valencia. Estudio, EC 2001-03, IVIE. Valencia.

Beraza, J. M., \& Rodríguez, A. (2010). Factores determinantes de la utilización de las spin-offs como mecanismo de transferencia de conocimiento en las universidades. Investigaciones Europeas de Dirección y Economía de la Empresa, 16(2), 115-135. doi:10.1016/\$11352523(12)60115-4
Bozeman, B., Rimes, H., \& Youtie, J. (2015). The evolving state-ofthe-art in technology transfer research: Revisiting the contingent effectiveness model. Research Policy, 44(1), 34-49. doi:10.1016/j.respol.2014.06.008

Bradley, S., Hayter, C., \& Link, A. (2013). Models and Methods of University Technology Transfer. Foundations and Trends ${ }^{\circledast}$ in Entrepreneurship, 9(6), 571-650. doi:10.1561/0300000048

Carayannis, E., \& Campbell, D. (2012). Mode 3 Knowledge Production in Quadruple Helix Innovation Systems.

Etzkowitz, H. (2017). Innovation Lodestar: The entrepreneurial university in a stellar knowledge firmament. Technological Forecasting and Social Change, 123, 122-129. doi:10.1016/j.techfore.2016.04.026

Fernández, S., Otero, L., Rodeiro, D., \& Rodríguez, A. (2009). Determinantes de la capacidad de las universidades para desarrollar patentes. Revista de la educación superior, 38, 7-30.

Foltz, J., Barham, B., \& Kim, K. (2000). Universities and agricultural biotechnology patent production. Agribusiness, 16(1), 82-95. doi:10.1002/(sici)1520-6297(200024)16:1<82::Aid-agr7>3.0.Co;2-v

Heinzl, J., Kor, A.-L., Orange, G., \& Kaufmann, H. R. (2013). Technology transfer model for Austrian higher education institutions. The Journal of Technology Transfer, 38(5), 607-640. doi:10.1007/s10961012-9258-7

Iglesias, P., Jambrino, C., \& Peñafiel, A. (2012). Caracterización de las Spin-Off universitarias como mecanismo de transferencia de tecnología a través de un análisis clúster. Revista Europea de Dirección y Economía de la Empresa, 21(3), 240-254. doi:10.1016/j.redee.2012.05.004

Kochenkova, A., Grimaldi, R., \& Munari, F. (2016). Public policy measures in support of knowledge transfer activities: a review of academic literature. The Journal of Technology Transfer, 41(3), 407-429. doi:10.1007/s10961-015-9416-9

Lederman, D., \& Maloney, W. (2014). Innovación en Chile: ¿Dónde Estamos?, (ISSN 0717-9987).

López, M., Mejía, J., \& Schmal, R. (2006). Un Acercamiento al Concepto de la Transferencia de Tecnología en las Universidades y sus Diferentes Manifestaciones. Panorama Socioeconómico.

Menezes, F., Liska, G., Cirillo, M., \& Vivanco, M. (2017). Data classification with binary response through the Boosting algorithm and logistic regression. Expert Systems with Applications, 69, 62-73. doi:10.1016/j.eswa.2016.08.014

Perkmann, M., Tartari, V., McKelvey, M., Autio, E., Broström, A., D'Este, P., . . S Sobrero, M. (2013). Academic engagement and commercialisation: A review of the literature on university-industry relations. Research Policy, 42(2), 423-442. doi:10.1016/j.respol.2012.09.007 
Peters, M. (1989). Techno-Science, Rationality, and the University: Lyotard on the "Postmodern Condition"1. Educational Theory, 39(2), 93-105. doi:10.1111/j.1741-5446.1989.40000.x

Rodriguez, J., \& Casani, F. (2007). La transferencia de tecnología en España: diagnóstico y perspectivas. Economía industrial, ISSN 04222784, № 366.

Rojo, J. M. (2007). Regresión con variable dependiente cualitativa. In: Laboratorio de estadística.

Siegel, D., Waldman, D., Atwater, L., \& Link, A. (2004). Toward a model of the effective transfer of scientific knowledge from academicians to practitioners: qualitative evidence from the commercialization of university technologies. Journal of Engineering and Technology Management, 21(1), 115-142. doi:10.1016/j.jengtecman.2003.12.006

Siegel, D., Waldman, D., \& Link, A. (2003). Assessing the impact of organizational practices on the relative productivity of university technology transfer offices: an exploratory study. Research Policy, 32(1), 27-48. doi:10.1016/S0048-7333(01)00196-2
Sternberg, R. (2014). Success factors of university-spin-offs: Regional government support programs versus regional environment. Technovation, 34(3), 137-148. doi:10.1016/j.technovation.2013.11.003

Thursby, J., Jensen, R., \& Thursby, M. (2001). Objectives, Characteristics and Outcomes of University Licensing: A Survey of Major U.S. Universities. The Journal of Technology Transfer, 26(1), 59-72. doi:10.1023/A:1007884111883

Van Norman, G., \& Eisenkot, R. (2017). Technology Transfer: From the Research Bench to Commercialization: Part 1: Intellectual Property Rights-Basics of Patents and Copyrights. JACC: Basic to Translational Science, 2(1), 85-97. doi:10.1016/j.jacbts.2017.01.003

Wright, M., Clarysse, B., Lockett, A., \& Knockaert, M. (2008). Mid-range universities' linkages with industry: Knowledge types and the role of intermediaries. Research Policy, 37(8), 1205-1223. doi:10.1016/j.respol.2008.04.021

Zuñiga, P., \& Correa, P. (2013). Technology Transfer from Public Research Organizations: Concepts, Markets, and Institutional Failures. In: The Innovation Policy Platform. 